\title{
DISTRIBUIÇÃO DE FERTILIZANTES A LANÇO EM FUNÇÃO DA FRAÇÃO
} GRANULOMÉTRICA

Étore Francisco Reynaldo ${ }^{1}$, Thiago Martins Machado ${ }^{2}$, Leandro Taubinger ${ }^{3}$, Dionathan de Quadros ${ }^{4}$ Sascha Ronny Scwartz ${ }^{5}$

${ }^{1}$ Pesquisador da Fundação Agrária de Pesquisa Agropecuária (etorereynaldo@hotmail.com) - Entre Rios - PR - Brasil

${ }^{2}$ Professor Doutor do Instituto de Ciências Agrárias e Ambientais da Universidade

Federal de Mato Grosso - Campus Sinop - Sinop - MT - Brasil

${ }^{3}$ Mestrando em Produção Vegetal na Unicentro - Guarapuava - PR - Brasil

${ }^{4}$ Engenheiro Agrônomo e Auxiliar técnico da Fundação Agrária de Pesquisa Agropecuária - Entre Rios - PR - Brasil

${ }^{5}$ Trainee de processos, Cooperativa Agrária Agroindustrial - Entre Rios - PR - Brasil

Recebido em: 08/04/2016 - Aprovado em: 30/05/2016 - Publicado em: 20/06/2016 DOI: 10.18677/Enciclopedia_Biosfera_2016_043

\begin{abstract}
RESUMO
A qualidade e a uniformidade dos fertilizantes, são fatores determinantes para o estabelecimento da largura efetiva de trabalho de equipamentos de distribuição de fertilizantes a lanço. Sendo assim, objetivou-se com o presente trabalho, avaliar o comportamento da distribuição transversal de fertilizantes em função de diferentes fontes. O distribuidor utilizado possui mecanismo dosador gravimétrico e mecanismo distribuidor centrífugo com dois discos horizontais. O equipamento de distribuição foi acoplado a um pulverizador autopropelido com engate traseiro de três pontos com quadro móvel e barras frontais. Foram utilizados dois fertilizantes nitrogenados: Uréia Nacional (45-00-00), denominado de Ua e Yara Bela (27-00-00) denominado de Ub. Cada tratamento consistiu em três passadas sobre coletores padronizados, sendo que após as passadas, o produto foi coletado e pesados em balança eletrônica e analisada a granulometria. Os dados obtidos foram analisados em planilha eletrônica "Adulanço" onde se calculou o coeficiente de variação (CV) em função da largura efetiva de trabalho simulada em 30 metros. O fertilizante Ub apresentou valores de (CV) abaixo do limite na largura pré-estabelecida e também obteve qualidade no perfil de distribuição, possibilitando o trabalho em faixas maiores, devido a maior uniformidade granulométrica e maior concentração dos grânulos nas classes maiores.
\end{abstract}

PALAVRAS-CHAVE: Adubador, máquinas agrícolas, uréia

\section{FERTILIZER DISTRIBUTION TO HAUL FOR EACH FRACTION GRADING}

\begin{abstract}
The quality and uniformity of fertilizers, are determining factors for setting the effective width of fertilizer distribution equipment. Therefore, this study objective to evaluate the behavior of the transverse distribution of fertilizers according to different
\end{abstract}


sources. It is the distributor used with gravimetric metering system and mechanism centrifugal distributor with two horizontal discs. The distribution equipment is coupled to a self-propelled sprayer with front bars, possessing rear three-point hitch with mobile frame. Urea fertilizers were used two urea (45-00-00) and Ub Yara Bela (2700-00), each treatment consisted of three standard collectors passed on, and after the past, the product was collected and weighed on an electronic scale and analyzing the particle size. The data were analyzed in a spreadsheet "Adulanço" which was calculated the coefficient of variation (CV) as a function of the simulated effective width of 30 meters. Fertilizer Ub presented values of the coefficient of variation below the threshold at predetermined width and also in the quality of cross distribution profile, enabling the work in larger groups due to more uniform particle size and greater concentration of beads in the larger classes.

KEYWORDS: farm machinery, fertilizer, urea

\section{INTRODUÇÃO}

Devido a busca cada vez maior pela produtividade e lucratividade, a qualidade do fertilizante ligada a uniformidade e granulometria, tem uma importância fundamental e determinante na produtividade das culturas. A aplicação de fertilizantes é um processo essencial para a manutenção de níveis de nutrientes no solo adequados ao desenvolvimento das culturas. No entanto, a aplicação destes produtos químicos sem critério origina problemas ambientais e tem custos econômicos e energéticos (SERRANO et al., 2011; SERRANO et al., 2014). Uma adubação desequilibrada pode resultar em perdas econômicas e ambientais, ressaltando-se que a maioria dos insumos é derivada de fontes não renováveis, cuja crescente demanda e escassez de reservas tem elevado os custos ao longo dos anos (SANTOS et al., 2012).

O correto uso de equipamentos denominados de distribuidores de corretivos e fertilizantes é de extrema importância para se atingir as metas de produtividade desejadas (BAIO et al., 2012). Segundo PORTELLA \& BATISTA (2012), no Brasil os ensaios de distribuidores de fertilizantes são feitos da forma convencional, onde utilizam-se bandejas para coletar o produto e posteriormente depositadas em um recipiente para a pesagem em balança eletrônica. No entanto, as máquinas geralmente utilizadas, têm a característica principal, de dependência com relação a uniformidade do produto a ser aplicado. Para avaliação de regularidade de distribuição transversal, utiliza-se a norma ASABE (2006), sendo a analise efetuada pelo coeficiente de variação da distribuição dos fertilizantes.

Com o crescimento da utilização dos conceitos de agricultura de precisão associados à aplicação de insumos a taxas variáveis, surge também à necessidade de se avaliar o comportamento da distribuição transversal de fertilizantes. Desta forma, objetivou-se com a realização deste trabalho, avaliar o comportamento da distribuição transversal de fertilizantes em função de diferentes fontes.

\section{MATERIAL E MÉTODOS}

O experimento foi realizado na Fazenda dos Porcos, localizada no município de Goioxim - PR, nas seguintes coordenadas geográficas: Latitude -25'16' e Longitude -51\%6'.

O equipamento utilizado no comparativo de perfil de distribuição transversal de fertilizante foi o modelo Accura 1600 (Figura 1) marca Kuhn ${ }^{\circledR}$. O distribuidor é equipado com reservatório tipo pirâmide invertida e mecanismo dosador gravimétrico auxiliado por agitador mecânico. O mecanismo distribuidor é centrífugo com dois 
discos horizontais, modelo S8. O equipamento de distribuição foi acoplado ao pulverizador autopropelido $\mathrm{Pla}^{\circledR}$, modelo: $\mathrm{H} 3000 \mathrm{com}$ barras frontais (Figura 1). $\mathrm{O}$ espécime possui engate traseiro de três pontos com quadro móvel, possibilitando o trabalho (distribuição) em diferentes alturas.

A velocidade do distribuidor autopropelido, foi de $8 \mathrm{~km} \mathrm{~h} \mathrm{~h}^{-1}$, conforme LAGHARI et al. (2014).

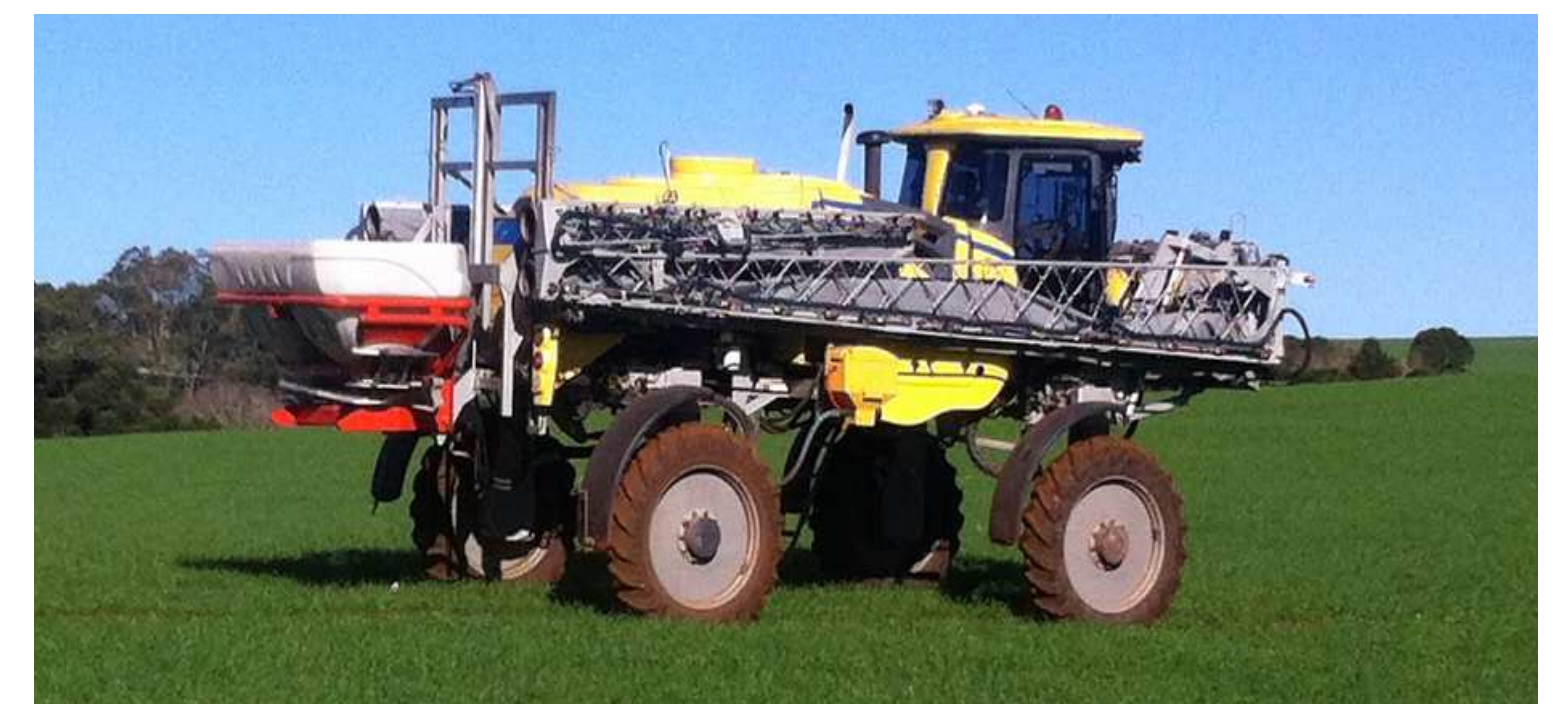

FIGURA 1 - Pulverizador autopropelido e distribuidor de fertilizantes centrifugo montado.

Fonte: (REYNALDO, 2015)

A velocidade do vento foi monitorada no decorrer de cada passada com o auxílio de um anemômetro digital portátil. Os fertilizantes utilizados na comparação foram: Uréia Nacional (45-00-00), denominado de Ua e YaraBela (27-00-00) denominado de Ub.

A determinação da granulometria geral se deu com a coleta de uma amostra de $250 \mathrm{~g}$ de fertilizante antes que o mesmo passasse pelos mecanismos dosadores. As amostras foram fracionadas em partes, a fim de se obter 100 gramas de produto. Após o fracionamento, o fertilizante, foi colocado em um agitador automático. Foram utilizadas as peneiras com malhas de $4 ; 2,8 ; 2 ; 1,7 ; 1 ; 0,5$ e fundo das peneiras, acopladas, o período de agitação das amostras foi de 10 minutos. Após o processo de agitação as quantidades retidas em cada peneira foram pesadas e os valores convertidos em porcentagens, para as devidas comparações entre as peneiras, conforme recomendações do MAPA (2013).

As determinações do teor de água, densidade, densidade específica e ângulo de repouso seguiram a metodologia e as análises proposta por Reynaldo \& Gamero (2015). As avaliações para distribuição transversal foram desenvolvidas de acordo com a norma ASABE (2006). Os coletores foram alinhados paralelamente no campo, de forma transversal a distribuição do material.

A vazão do mecanismo dosador foi determinada com base no manual do fabricante, conferidas e adequadas para a dose de $200 \mathrm{~kg} \mathrm{ha}^{-1}$. Foi realizada a análise da vazão com o distribuidor com meia carga, sendo o produto coletado considerando-se a totalização dos dois mecanismos dosadores. A altura utilizada no ensaio foi de $1150 \mathrm{~mm}$ em relação ao solo. 
Avaliações antecedentes foram realizadas para a determinação do ponto de queda do produto no disco horizontal, partindo do especificado pelo manual do fabricante fez-se um acima e um abaixo. O melhor ponto de queda foi na posição 5,0 para ambos os fertilizantes avaliados.

TABELA 1- Tratamentos e suas interações avaliadas no ensaio.

\begin{tabular}{cccc}
\hline$N^{\circ}$ Trat. & Produto & $\begin{array}{c}\text { Dose } \\
\left(\mathrm{kg} \mathrm{ha}^{-1}\right)\end{array}$ & Altura de aplicação $(\mathrm{m})$ \\
\hline 1 & Ua & 200 & 1,15 \\
2 & Ub & 200 & \\
\hline
\end{tabular}

Os coletores utilizados possuíam dimensões padronizadas de $500 \times 500 \times 150$ $\mathrm{mm}$ respectivamente a comprimento, largura e profundidade. Dentro de cada coletor foi inserido uma grade de polietileno $(75 \times 75 \mathrm{~mm}$ de abertura de alvéolo e $60 \mathrm{~mm}$ de profundidade) para que fosse evitado o ricocheteamento do material, conforme recomenda a ASABE (2006). O ensaio foi conduzido com a regulagem de largura efetiva fixa de 30 metros. Para tanto utilizou-se 136 coletores, totalizando 70 metros sobre a fila de coletores dispostos lado a lado entre si. A velocidade de deslocamento do conjunto foi de $8 \mathrm{~km} \mathrm{~h}^{-1}$, sendo que o deslocamento se deu perpendicularmente ao eixo de disposição dos coletores, os quais tinham espaços livres de 2 coletores nos locais por onde se deslocava o rodado.

Cada tratamento consistiu em três passadas sobre os coletores, sendo que após as passadas, o produto foi coletado em copos plásticos enumerados e pesados em balança eletrônica com resolução de 0,01 grama. Os dados obtidos foram analisados em planilha eletrônica "Adulanço versão 3.0" MOLIN (2009) de acordo com metodologia proposta por MOLIN et al. (2009), onde se calculou o (CV) em função da largura efetiva simulada de 30 metros. O Coeficiente de variação é uma medida de desuniformidade da dose aplicada com a sobreposição de passadas adjacentes. A faixa efetiva de trabalho foi considerada como aceitável para valores de CV abaixo do pré-estabelecido de 20\%. GOMES (2009) considera um CV de 10 a $20 \%$ como um valor médio.

Devido ao bom desempenho do produto Ub na largura efetiva simulada de 30 metros, foi realizada também uma simulação de aumento da largura efetiva de trabalho, para verificar qual seria essa largura quando se assume trabalhar com CV no limite, ou seja, de $20 \%$.

\section{RESULTADOS E DISCUSSÃO}

A velocidade média mensurada do vento no decorrer do ensaio foi de $1,1 \mathrm{~m} \mathrm{~s}^{-1}$, valor este dentro do limite recomendado, que é de 2,22 $\mathrm{m} \mathrm{s}^{-1}$ conforme ASABE (2006), portanto não havendo influência de meios externos nos resultados. Na Tabela 2 tem-se a granulometria dos fertilizantes utilizados no ensaio de distribuição transversal entre os equipamentos.

Aproximadamente $64 \%$ do fertilizante Ub apresentou fração de grânulos com diâmetros superior a 2,8mm, 33\% de grânulos com diâmetros maiores que $4,0 \mathrm{~mm}$. Para a Ua, a fração de grânulos com diâmetros superior a 2,8mm não apresentou grande diferença. No entanto, aproximadamente 37\% dos grânulos apresentaram diâmetro entre 2,0 e 2,8mm. Em experimento semelhante REYNALDO et al. (2016) concluiram que $79 \%$ do fertilizante de ureia cloretada linha Premium apresentou fração de grânulos com diâmetros superior a 2,8 mm, sendo 12 \% de grânulos com ENCICLOPÉDIA BIOSFERA, Centro Científico Conhecer - Goiânia, v.13 n.23; p. 490 
diâmetros maiores que $4,0 \mathrm{~mm}$, e o fertilizante uréia cloretada comum, aproximadamente $63 \%$ dos grânulos apresentavam diâmetros superior a $2,8 \mathrm{~mm}$, sendo $3 \%$ de grânulos com diâmetros maiores que $4,0 \mathrm{~mm}$.

TABELA 2 - Granulometria dos fertilizantes utilizados no ensaio.

\begin{tabular}{ccc}
\hline \multirow{2}{*}{ Peneira } & \multicolumn{2}{c}{ Frações granulométricas \% } \\
\cline { 2 - 3 } & Ua & Ub \\
\hline $4,0 \mathrm{~mm}$ & 2,72 & 33,00 \\
$>2,8 \mathrm{~mm}$ & 59,23 & 64,20 \\
$>2,0 \mathrm{~mm}$ & 36,69 & 2,79 \\
$>1,7 \mathrm{~mm}$ & 0,96 & 0,04 \\
$>1,0 \mathrm{~mm}$ & 0,35 & 0,02 \\
$>0,5 \mathrm{~mm}$ & 0,03 & 0,00 \\
Pó & 0,03 & 0,00 \\
\hline
\end{tabular}

Partículas com granulometria diferente apresentam comportamento de balística diferente. Quanto maior a partícula, maior a distância alcançada para uma mesma força de lançamento. Sendo assim, o fertilizante Ub se mostrou mais vantajoso que a Ua, devido a possibilidade conforme resultados, do aumento de largura efetiva de trabalho, e consequentemente aumento de rendimento operacional.

A desuniformidade dos fertilizantes tem forte relação com a qualidade durante o processamento, influenciando na quebra de grânulos devido à baixa dureza MOLIN et al. (2009). Outra possível explicação pode estar relacionada ao transporte dos fertilizantes, onde os mesmos são expostos a segregação das partículas devido a vibração.

Na Figura 2 é apresentado o gráfico de perfil de distribuição transversal obtido com uso de Ua. A Ua, apresentou uma distribuição com o formato piramidal e maior volume de concentração no centro.

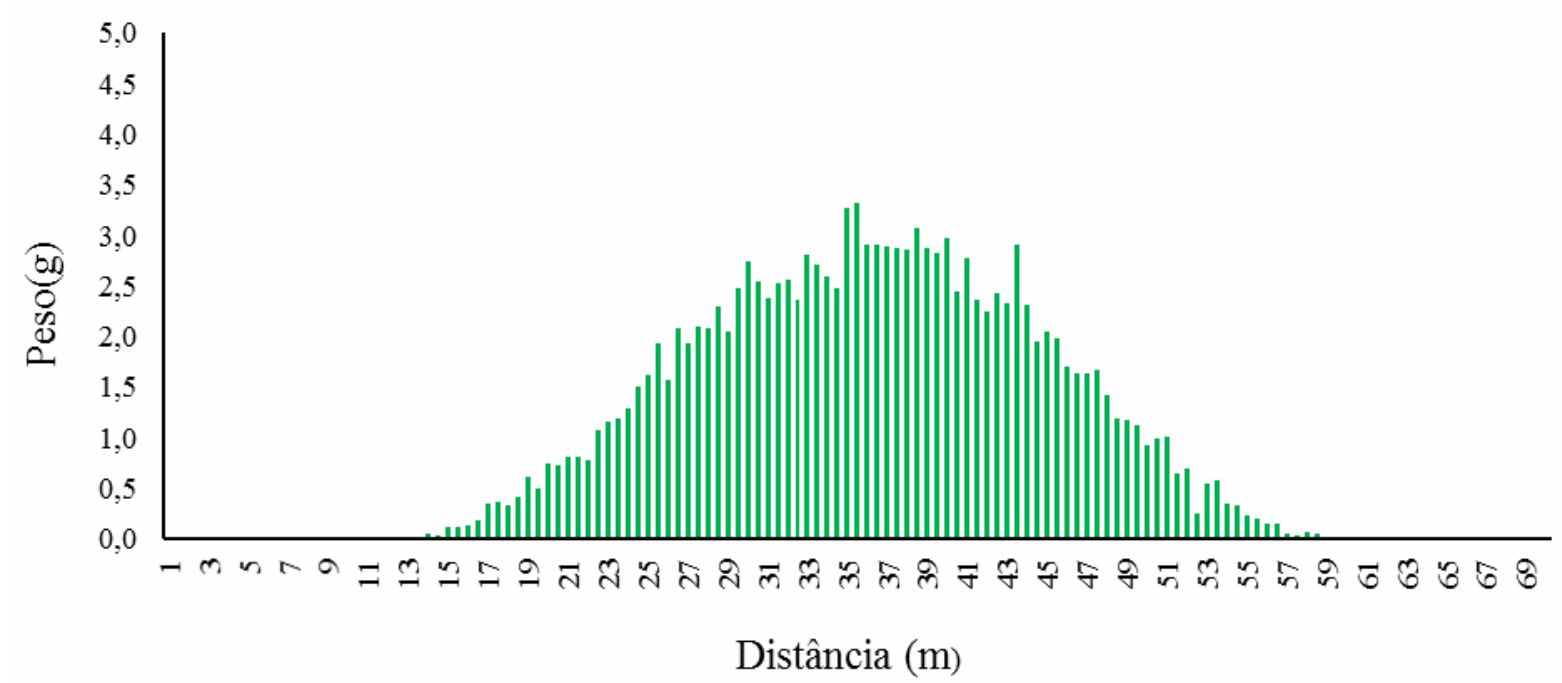

FIGURA 2 - Perfil de distribuição transversal obtido com uso de Ua. 
Na Figura 3 é apresentado o perfil de distribuição transversal obtido com uso de Ub para a largura de trabalho definida. Nota-se que este material apresentou melhor distribuição que a Ua, visto que houve distribuição homogenia ao longo da faixa de coleta (extremos).

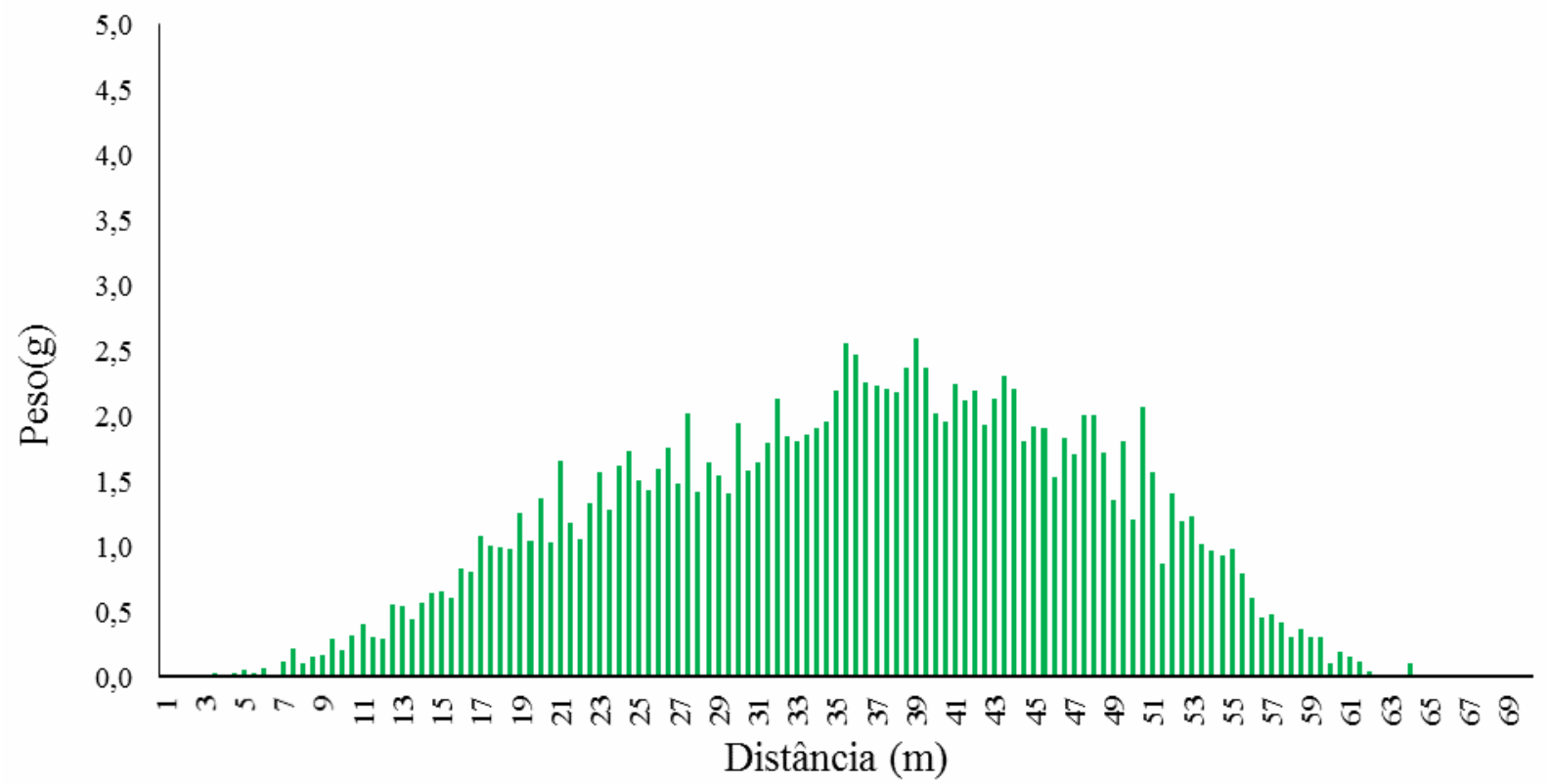

FIGURA 3 - Gráfico de perfil de distribuição transversal obtido com uso de Ub.

Na Tabela 3 é apresentado o CV para largura efetiva de 30 metros para os diferentes fertilizantes avaliados. O CV\% se manteve abaixo do limite de $20 \%$ para o produto Ub, para os três modos de percurso simulados, apresentando um desempenho superior à Ua, apresentou CV acima do limite especificado para os modos de percurso alternado esquerdo e contínuo.

TABELA 3 - Coeficiente de variação nos diferentes fertilizantes avaliados, na largura efetiva de $30 \mathrm{~m}$ e largura efetiva de trabalho no máximo CV aceitável.

\begin{tabular}{lcccc}
\hline \multirow{2}{*}{ Modo de Percurso } & \multicolumn{3}{c}{ Coeficiente de Variação (\%) } \\
\cline { 2 - 5 } & \multicolumn{3}{c}{ Ua } & \multicolumn{3}{c}{ Ub } \\
\hline Alternado Direito & 20,28 & & 13,68 & \\
Alternado Esquerdo & 23,60 & 20,00 & 12,24 & 20,00 \\
Contínuo & 21,98 & & 15,14 & \\
\hline Largura efetiva de trabalho $(\mathrm{m})$ & 30 & 29,40 & 30 & 40,88 \\
\hline
\end{tabular}

Também na Tabela 3 é apresentada a largura de trabalho obtida quando se assume trabalhar com CV no limite, ou seja, em torno de $20 \%$. Assim é possível trabalhar com largura de 40,88 m para o fertilizante Ub, apresentando aumento de 20 a $40 \%$ de rendimento na operação de distribuição de fertilizante sólido a lanço, dependendo das condições de eficiência operacional e de tamanho e formato da área a ser trabalhada. Em avaliação da distribuição com diferentes fertilizantes, marcas e modelos de distribuidores de fertilizantes, avaliando em pátio e em culturas, considerando um CV de $20 \%$ BAIO et al. (2012) encontraram larguras 
efetivas de trabalho variando de 10,92 a $13,17 \mathrm{~m}$, portanto, muito inferiores as obtidas nas avaliações.

Fica evidenciado através das simulações com as diferentes fontes de fertilizantes o quanto a eficiência das máquinas aplicadoras é influenciada pela qualidade dos fertilizantes.

\section{CONCLUSÕES}

A qualidade dos fertilizantes tem influência direta na qualidade de distribuição dos distribuidores de fertilizantes a lanço.

O fertilizante Ub apresentou valores do CV abaixo do limite na largura préestabelecida.

O fertilizante Ub apresentou maior qualidade no perfil de distribuição, possibilitando o trabalho em maiores faixas.

A ureia nacional apresentou CV acima do estabelecido para a largura de trabalho.

O desempenho superior do fertilizante Ub se deu provavelmente pela maior concentração dos grânulos nas classes maiores.

\section{REFERÊNCIAS}

ASABE. ASAE S341.3. Procedure for measuring distribution uniformity and calibrating granular broadcast spreaders. St Joseph: ASABE Standards, 215-218, 2006.

BAIO, F. H. R.; MOLIN, J. M.; LEAL, A. J. F. Avaliação da distribuição transversal de adubos sólidos aplicados em culturas anuais. Biosci. J. Uberlândia, v.28, p. 527536. 2012.

GOMES, P. F. Curso de estatística experimental.15 ed. Piracicaba: Nobel, 2009. $451 \mathrm{p}$.

LAGHARI, M.; LAGHARI, N.; SHAH, A. R.; CHANDIO, F. A. Calibration and Performance of Tractor Mounted Rotary Fertilizer Spreader. International Journal of Advanced Research, v. 2, p.839-846. 2014.

MAPA. Ministério da Agricultura Pecuária e Abastecimento. Manual de métodos analíticos oficiais para fertilizantes e corretivos. 150p, 2013. Disponível em:<http://sistemasweb.agricultura.gov.br/arquivosislegis/anexos/arquivos/1204363. PDF > Acesso em: 10 março 2016.

MOLIN, J.P. Adulanço 3.0: montagem do teste de campo, Manual de uso passoa-passo, Análise de resultados. Piracicaba: USP/ESALQ. Projeto AP. 20p, 2009.

MOLIN, J. P.; MACHADO, T. M.; MAGALHAES, R. P.; FAULIN, G. D. C. Segregação de fertilizantes aplicados a lanço. Engenharia Agrícola. v.29, pp. 614-622, 2009. Disponível em: < http://dx.doi.org/10.1590/S0100-69162009000400011>. Acesso em: 10 março 2016. Doi: 10.1590/S0100-69162009000400011.

PORTELLA, J. A.; BATISTA, D. G. Teste eletrônico. Cultivar Máquinas, Não Me Toque. n. 118, p.12-14. 2012. 
REYNALDO, E. F.; GAMERO, C. A. Avaliação de mecanismos dosadores de fertilizantes helicoidais em ângulo de nivelamento longitudinal e transversal. Energia na agricultura, Botucatu, v.30, p.125-136. 2015.

REYNALDO, E. F.; MACHADO, T. M.; TAUBINGER, L.; QUADROS, D. Distribuição de fertilizantes a lanço em função da qualidade do insumo. Energia na Agricultura, v. 31, p. 24-30, 2016. Disponível em: <http://dx.doi.org/10.17224/EnergAgric.2016v31n1p24-30 >. Acesso em: 10 maio 2016. Doi: 10.17224/EnergAgric.2016v31n1p24-30.

SANTOS, F. C.; FILHO, M. R. A.; NOVAIS, R. F.; FERREIRA, G. B.; CARVALHO, M. C. S.; FILHO, J. L. S. Fontes, doses e formas de aplicação de fósforo para o algodoeiro no cerrado da Bahia. Revista Ceres, Viçosa, v. 59, p.537-543. 2012.

SERRANO, J.; PEÇA, J.; SILVA, J. M.; SHAHIDIAN, S. Aplicação de fertilizantes: tecnologia, eficiência energética e ambiente. Revista de Ciências Agrárias, v.37, p.270-279. 2014.

SERRANO, J. M.; PEÇA, J.O. E SHAHIDIAN, S. B. Evaluation of variable rate technology for fertilizer application in permanent pastures. Journal of Agricultural Science and Technology, v.1, p.489-499. 2011. 